\title{
ALTERAÇÕES CARDÍACAS NA INFECÇÃO POR COVID-19
}

\author{
HEART CHANGES IN COVID-I9 INFECTION
}

\author{
Andresa Moura de Oliveira ${ }^{1}$ \\ Bárbara Giovana Rodrigues dos Santos ${ }^{2}$ \\ Kethellen Jade dos Reis Martins Gomes ${ }^{3}$ \\ Letícia Ketelly Soares da Rocha ${ }^{4}$ \\ Victoria Maria Alves Arruda ${ }^{5}$ \\ Lorran Miranda Andrade de Freitas ${ }^{6}$
}

RESUMO: Um novo patógeno se alastrou em Wuhan na China em dezembro de 2019, causando sintomas similares à pneumonia, sendo identificado posteriormente como SARS CoV-2, causador da doença COVID-ı́. Logo em seguida, a Organização Mundial da Saúde (OMS) o declarou como uma doença de emergência internacional de alto risco, uma pandemia. Dentre as diversas complicações causadas pela COVID-I9, as cardíacas são pouco mencionadas, entretanto representam um grande risco aos infectados, com destaque a indivíduos idosos e com comorbidades cardíacas. Assim, o estudo sobre as consequências da COVID-I9 nas complicações cardíacas, com ênfase nas alterações dos exames laboratoriais pode auxiliar no diagnóstico e tratamento de lesões cardiovasculares decorridas pelo SARS$\mathrm{CoV}-2$, melhorando o prognóstico do paciente. Foram utilizados artigos disponíveis na literatura online como base para construção do artigo, sendo observados os dados, a literatura relata que pelo menos $8 \%$ dos pacientes diagnosticados com COVID-i9 desenvolveu uma lesão cardíaca aguda, aumentando o risco de morte em duas vezes. Através da utilização de biomarcadores como a troponina ultrassensível e Dímero-D, que apresentam elevação em pacientes COVID-ı9 com lesão cardiovascular, o diagnóstico precoce do desenvolvimento de lesões pode ser obtido, aumentando as chances de sobrevivência e melhorando o prognóstico do paciente.

Palavras-chave: Covid-ıو. Manifestações. Cardiovasculares. Biomarcadores.

ABSTRACT: A new pathogen spread in Wuhan, China in December 2019, causing symptoms similar to pneumonia, and was later identified as SARS-CoV-2, which causes

\footnotetext{
I Discente do curso Biomedicina da Faculdade Única de Ipatinga (FUNIP). $4^{\circ}$ Período. E-mail: andresa_moura_mgcf@hotmail.com

2 Discente do curso Biomedicina da Faculdade Única de Ipatinga (FUNIP). $4^{\circ}$ Período E-mail: barbaragiovana.17@hotmail.com

3 Discente do curso Biomedicina da Faculdade Única de Ipatinga (FUNIP). $4^{\circ}$ Período. E-mail: kethellenjader@hotmail.com

4 Discente do curso Biomedicina da Faculdade Única de Ipatinga (FUNIP). $4^{\circ}$ Período. E-mail: ketellyleticia46@gmail.com

5 Discente do curso Biomedicina da Faculdade Única de Ipatinga (FUNIP). $4^{\circ}$ Período. E-mail: vicarrudar2244@outlook.com

${ }^{6}$ Docente da Faculdade Única de Ipatinga (FUNIP). E-mail: lorrandamiranda@hotmail.com
} 
COVID-I9 disease. Soon after, the World Health Organization (WHO) declared it as a high-risk international emergency disease, a pandemic. Among the various complications caused by COVID-19, cardiac complications are rarely mentioned, however they represent a great risk to those infected, with emphasis on elderly individuals and with cardiac comorbidities. Thus, the study on the consequences of COVID-I9 on cardiac complications, with an emphasis on alterations in laboratory tests, can assist in the diagnosis and treatment of cardiovascular injuries caused by SARS-CoV-2, improving the patient's prognosis. Articles available in the online literature were used as a basis for the construction of the article, with data being observed, the literature reports that at least $8 \%$ of patients diagnosed with COVID-i9 developed an acute cardiac injury, increasing the risk of death twice. Through the use of biomarkers such as ultra-sensitive troponin and D-dimer, which are elevated in COVID-I9 patients with cardiovascular injury, an early diagnosis of the development of injuries can be obtained, increasing the chances of survival and improving the patient's prognosis.

Keywords: Covid-ı9. Manifestations. Cardiovascular. Biomarkers.

\section{INTRODUÇÃO}

Uma pneumonia de origem desconhecida acometia a população e se espalhava rapidamente, os primeiros casos surgiram em Wuhan na China e logo foram notificados em todo o mundo, sendo declarado pela Organização Mundial da Saúde como uma doença de emergência internacional de alto risco para a população. No Brasil, foi registrado o primeiro caso no estado de São Paulo em fevereiro, logo depois se estendendo para todo país, após estudos identificarem o genoma viral o patógeno causador foi classificado como SARSCoV-2, pertencente à família Betacoronavírus, causador da doença COVID-19 (MINISTÉRIO DA SAÚDE, 2020; OMS, 2020).

A sua principal manifestação é no trato respiratório, mas conforme os números de casos foram aumentando ficou evidente que ele pode afetar também outros órgãos como o coração, causando lesões cardiovasculares. Em um estudo com 1527 pacientes, demonstrou que pelo menos 8,o\% dos diagnosticados com COVID-ı9 desenvolve lesão cardíaca aguda, dobrando o risco de morte (CHEN et al., 2019; ZHENG et al., 2020).

Devido às complicações cardíacas associadas à infecção por COVID-19 o aumento foi significativo no número de mortes, portanto a análise do impacto da doença no sistema cardíaco com desfecho fatal para os pacientes, o risco de lesões pode ser identificado através de exames laboratoriais, utilizando para compreender os mecanismos patogênicos do vírus SARS-CoV-2 sobre as células cárdicas pode auxiliar na melhora do prognóstico, sobrevida 
e no desenvolvimento de um tratamento adequado para que tenha redução na taxa de mortalidade relacionada com essa manifestação (GUO et al., 2020; ZHENG et al., 2020).

O presente trabalho foi realizado por alunas da Faculdade Única de Ipatinga para o Projeto Interdisciplinar, como requisito parcial para aprovação no $4^{\circ}$ período. As principais matérias abordadas foram Patologia Geral para estudar os mecanismos causadores da COVID-ı9, Epidemiologia para o levantamento de dados da infecção pelo Sars-CoV-2, Genética para estudo do sequenciamento genético do vírus, Imunologia para estudo do mecanismo de defesa do organismo humano e Microbiologia para estudo estrutural do vírus.

\section{METODOLOGIA}

Trata- se de um estudo realizado de revisão bibliográfica, através da literatura online, disponível em artigos do Google Acadêmico e do Public Medline (PUBMED). As obras foram selecionadas nos idiomas português e inglês, utilizando as palavras-chave Covid-I9; Manifestações; Cardiovasculares; Biomarcadores, Manifestations; Cardiovascular; Biomarkers, sendo os critérios de inclusão artigos disponíveis na íntegra gratuitamente, publicada nos anos de 2019 e 2020, que contribuíssem para a execução do trabalho. Os critérios de exclusão foram artigos que não contribuíssem para o desenvolvimento do trabalho, relatos de casos isolados, ausência de critérios estatísticos e artigos com bloqueio monetário.

A pesquisa foi realizada usando títulos, resumo e assunto lidos pela íntegra. Depois de concluída a seleção das referências bibliográficas, os dados foram compilados no programa computacional Microsoft Office Word $e$ as informações analisadas correlacionadas aos parâmetros estudados. Os dados reunidos foram utilizados para uma análise descritiva de apresentação dissertativa.

\section{DESENVOLVIMENTO}

\subsection{Descrição da covid-ı9}

A COVID-rg leva a um quadro de Insuficiência Respiratória causada pelo novo vírus denominado SARS-CoV-2 iniciou em Wuhan, na China. Nas últimas duas décadas houve ainda duas epidemias causadas por coronavírus, sendo a Síndrome Respiratória Aguda Grave (SARS-CoV) em 2002 e à Síndrome Respiratória do Oriente Médio (MERS-CoV) 
em 2012. Dezembro de 2019 foi relatado um grupo de indivíduos com doença respiratória de origem desconhecida relacionado com mercado de frutos do mar. Entretanto em janeiro de 2020 a doença já tinha se espalhado e o número de casos relatados na China já ultrapassava 9 mil (DAWEI et al., 2020; GUO et al., 2020).

O vírus foi identificado em amostras de lavagem broncoalveolar, através de um sequenciamento genético pela técnica de reação em cadeia da polimerase quantitativa em tempo real e sequenciamento de alto rendimento, observaram-se características semelhantes pertencentes à família do coronavírus. A cepa $\beta-\mathrm{CoV}$ mostrou-se similar a outra síndrome respiratória derivada de morcegos, associando como principal fonte de transmissão para os humanos (PASTRIAN-SOTO, 2020; XIAOWEI et al., 2020).

\subsection{Estrutura e patogenicidade do SARS-COV-2}

A família do coronavírus se divide em quatro tipos de gêneros, sendo relatado na literatura o Alphacoronavirus, Betacoronavirus, Gammacoronavirus e Deltacoronavirus. Sendo que o SARS-CoV- 2 é pertencente ao grupo Betacoronavírus (PASTRIAN-SOTO, 2020). Através de estudos cientistas descobriram que o Sars-CoV-2 invade a célula pelo processo de endocitose, ele necessita da proteína $S$ contida em sua superfície que se liga ao receptor da célula hospedeira denominada enzima conversora angiotensina 2 (ECA2) (PASTRIANSOTO, 2020; XIAOWEI et al., 2020).

O SARS-CoV-2 possui em sua estrutura ácido ribonucleico (RNA) com sentido positivo, onde influência diretamente na síntese de proteínas, codificando as poliproteínas que formam o complexo viral, replicase, transcriptase, e codificando proteínas estruturais. Esse RNA positivo serve como um molde para produção de RNA com sentido negativo, que a partir dele sintetiza uma nova cópia do genoma original que irá migrar para um compartimento intermediário entre o complexo de Golgi e o reticulo endoplasmático. Nesse compartimento, o RNA positivo se associará as proteínas $S, M$ e E, que são proteínas estruturais e às proteínas acessórias, assim formando novas partículas virais. $O$ material genético do vírus é protegido através de um nucleocapsídeo e pela membrana externa. No qual o nucleocapsídeo possui uma proteína $\mathrm{N}$ que se associará ao genoma do vírus, que em seguida será fosforilada e inserida na bicamada lipídica do envelope externo, no envelope 
externo encontra-se as proteínas estruturais, como a proteína Spike(S), proteína de membrana(M) e a proteína de envelope (E) (PASTRIAN-SOTO, 2020).

Figura I- Estrutura do Sars-CoV-2

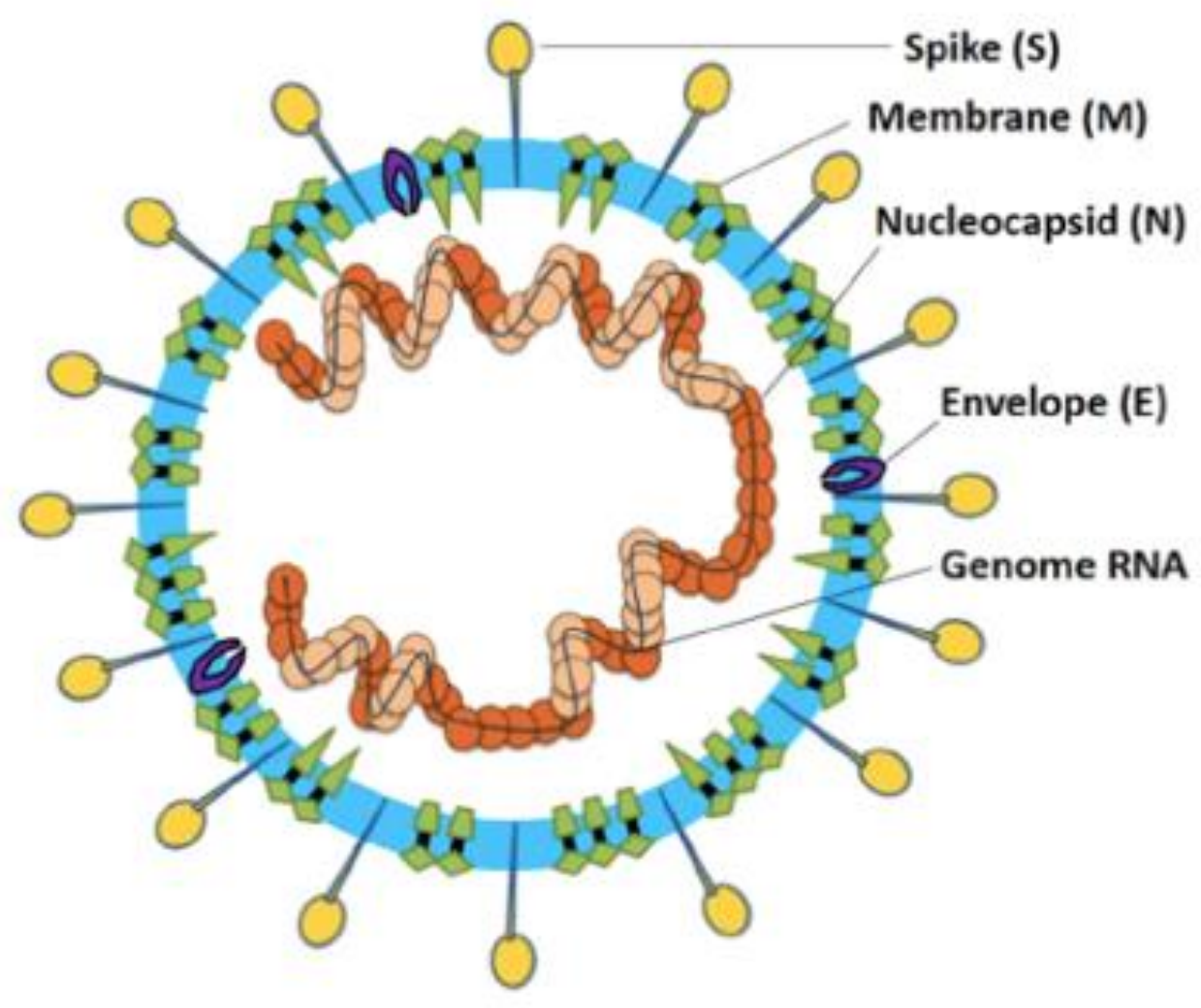

Fonte: MENEZES, 2020.

\subsection{Epidemiologia da COVID-19}

Desde os primeiros relatos no fim do ano de 2019 na China, o número de casos da COVID em Wuhan já chegava a mais de 80 mil, atingindo seu pico em fevereiro de 2020 . Janeiro de 2020, a Organização Mundial da Saúde (OMS) anunciou surto originado pelo novo coronavírus, elevando significativamente o número de casos, em março de 2020 foi declarado pandemia. Devido a sua facilidade de transmissão, em outubro de 2020 ultrapassam a 30 milhões de casos e aproximadamente I milhão de mortes mundialmente. Analisando os dados da Região das Américas há confirmação de aproximadamente ı6 milhões de casos e 559 mil mortes (MCINTOSH, 2020; OMS, 2020). 
No Brasil os números totais são de 5.380.635 casos e 156.903 mil mortes registradas, na região Sudeste o total de casos são de exatamente 1.884.738, Nordeste: 1.450.349, Norte: 18.430.980, Sul: 685.876 e Centro-Oeste: 674.720. Mundialmente o Brasil é o terceiro país com mais casos notificados, ficando abaixo apenas dos Estados Unidos (7.833.85I) e Índia (7.370.468) (OMS, 2020).

Gráfico I- Número de casos totais por regiões referentes à semana do dia 25 de outubro 2020.

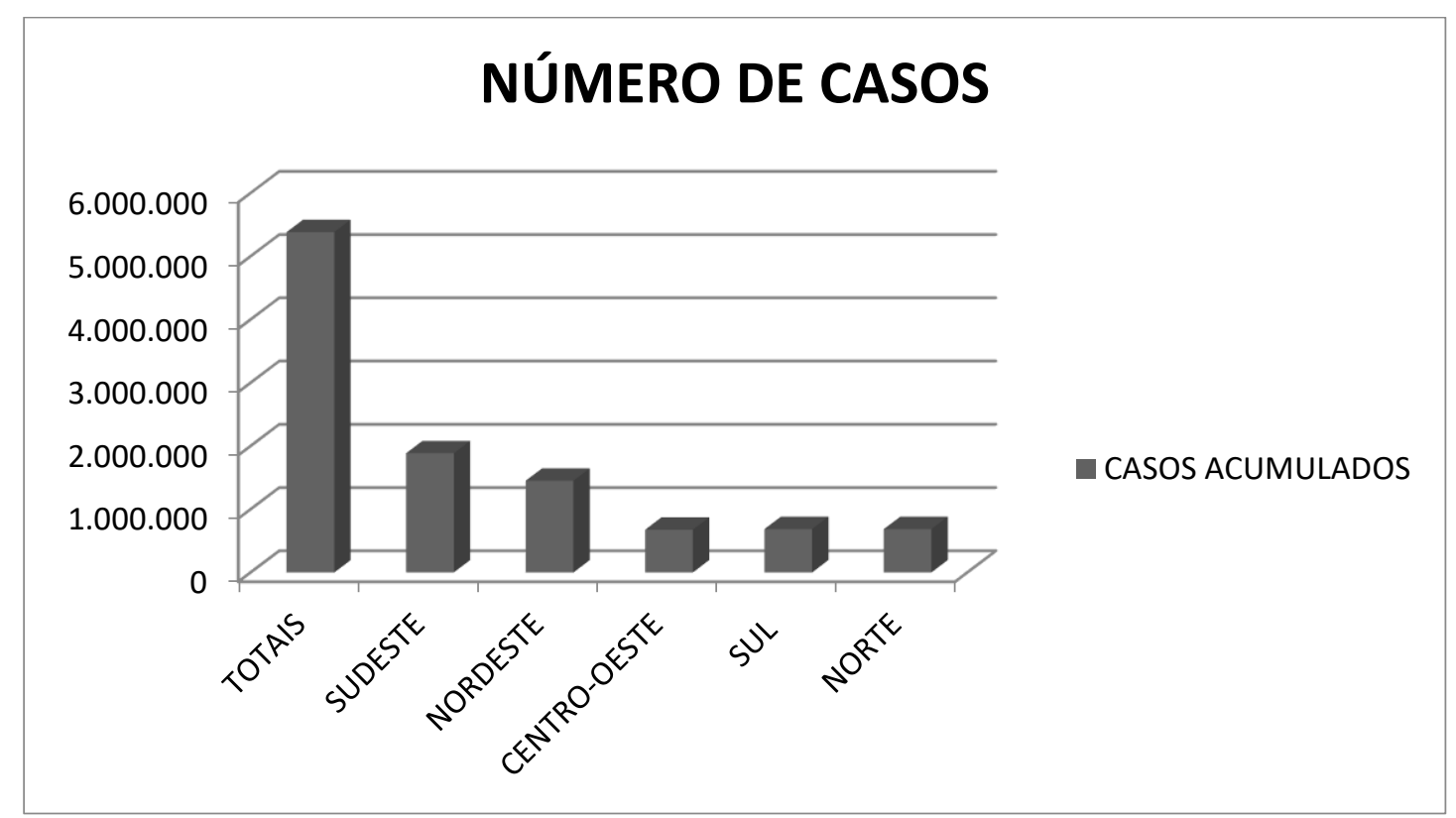

Fonte: AUTORES, 2020, com base nos dados das Secretarias Estaduais de Saúde. Brasil, 2020.

\subsection{Sintomas e transmissão}

O Sars-CoV-2 apresenta o período de incubação de aproximadamente I4 dias após a exposição, porém a maioria dos casos ocorre entre 4 a 5 dias. A disseminação acontece através de gotículas respiratórias de pessoa para pessoa, indivíduos que são assintomáticos também podem transmitir o vírus, além de objetos e superfícies contaminadas (MCINTOSH, 2020).

Segundo o Ministério da Saúde, 2020, devido a sua fácil transmissão pelo ar, são necessárias algumas formas de prevenção, sendo as principais lavar bem as mãos utilizando agua e sabão ou usar álcool a 70\%, evitar o contato com pessoas que estiverem tossindo ou espirrando, usar máscara de proteção facial, não ter contato a regiões como as dos olhos, boca 
e nariz. Caso houver manifestação de algum dos sintomas deve-se procurar o atendimento médico. Os sintomas podem variar de pessoa para pessoa, podem se apresentar de forma leve ou até mesmo de forma mais grave, principalmente para pessoas que possuem outras complicações. Os sintomas mais frequentes são febre, tosse e cansaço, porém pode haver outros sintomas como diarreia, dor de garganta, coriza, perda de paladar e olfato, entre outros. Cerca de $80 \%$ das pessoas infectas são assintomáticas ou oligo sintomáticos (MINISTÉRIO DA SAÚDE, 2020).

\subsection{Complicações cardiovasculares e COVID-19}

A COVID-ı9 está altamente relacionada à Insuficiência Respiratória, mas há também outras complicações como a doença cardiovascular, devido a sua alta carga inflamatória que pode induzir lesões no miocárdio, arritmias e miocardite. Além da manifestação comum da doença, comorbidades podem agravar o quadro clínico do paciente, como a hipertensão, diabetes e principalmente o fator idade, no qual é a forma mais grave da doença e a taxa de mortalidade concentrada em pacientes mais velhos. Indivíduos que já possuíam complicações cardíacas antes do surgimento da COVID-I9 estão mais suscetíveis à fatalidade, e naqueles que não possuíam histórico prévio houve o surgimento de danos cardíacos, podendo levar a complicações ainda maiores e irreversíveis, necessitando o encaminhamento para a unidade de tratamento intensivo (UTI) (DAWEI et al., 2019; MADJID et al., 2020; ZHENG et al., 2020).

Por ser uma doença relativamente nova os mecanismos fisiopatológicos do SARSCoV-2 em relação a sua ação no tecido cardiovascular vêm sendo discutidos, entretanto a hipóxia, tempestade de citosinas e a manifestação nos tecidos cardiovasculares são alguns dos fatores que podem estar relacionados diretamente a lesões no coração relatado na maioria das situações mais graves (DAWEI et al., 2019; ZHENG et al., 2020).

\subsection{Manifestações do SARS-CoV-2 nos tecidos cardiovasculares}

A relação entre o SARS-CoV-2 e os danos cardiovasculares está ligada a enzima conversora angiotensina 2, sendo altamente expressa em tecidos do sistema pulmonar e cardiovascular, estando envolvida no bom funcionamento do coração, além de ser relacionada a hipertensão e diabetes. Entretanto, o SARS-CoVs-2 utiliza a ECA2 como 
entrada na célula cardíaca através da ligação de sua espicula no receptor da ECA2, gerando lesão no miocárdio devido à invasão do vírus. Pacientes hipertensivos que fazem o uso de inibidores do sistema renina-angiotensina-aldosterona sofrem um aumento da síntese de ECA2, consequentemente sendo mais suscetíveis aos danos cardíacos (CHEN et al., 2019; GUZIK et al., 2020; ZHENG et al., 2020).

Figura 2- Invasão do Sars-CoV-2 na célula humana

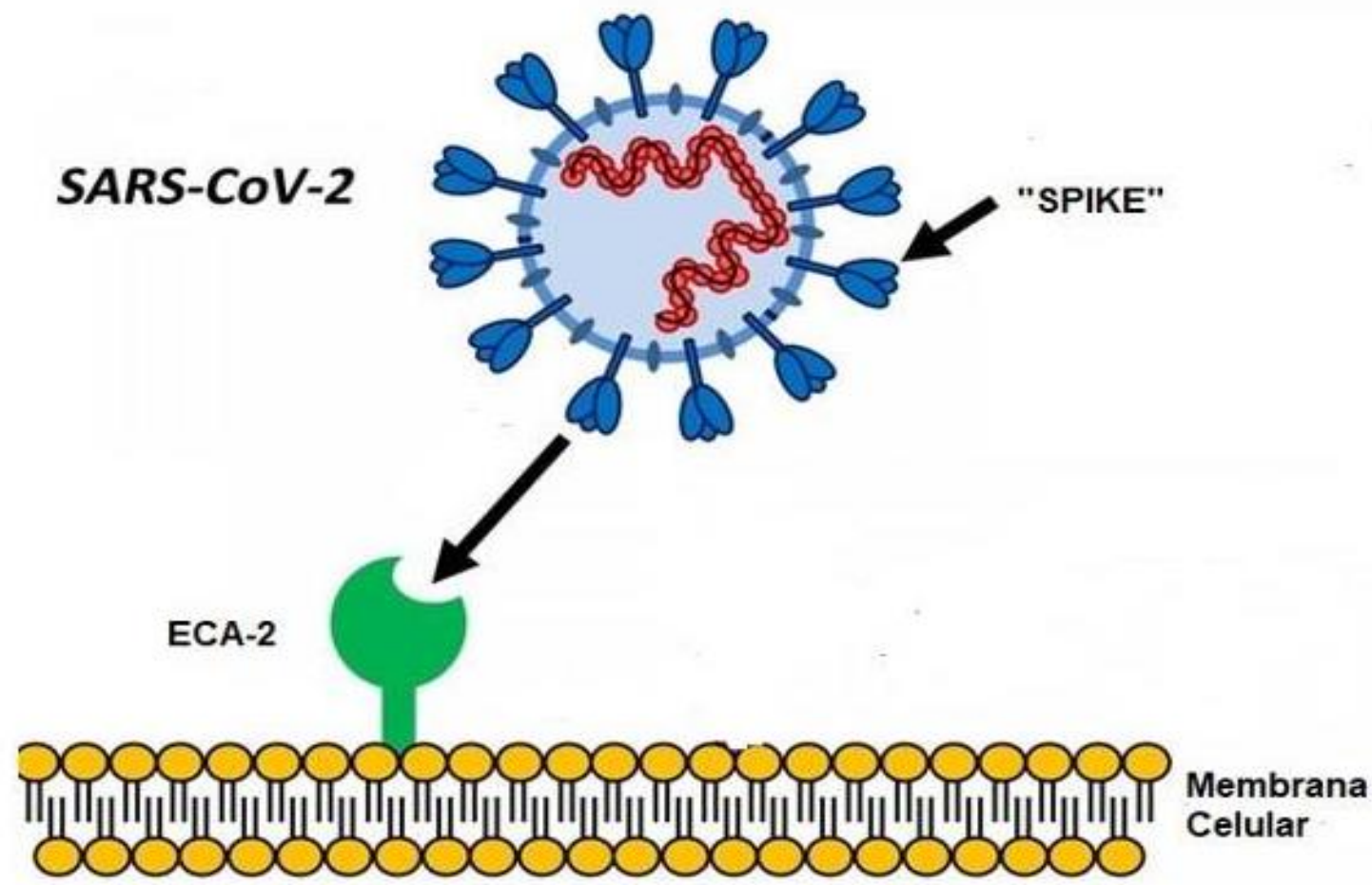

Fonte: SUAIDEN, 2020.

\subsection{Hipoxia}

A hipoxemia foi relatada em mais de $32 \%$ dos pacientes infectados pelo SARS-CoV2 em um estudo realizado em Huang, é uma condição onde ocorre falta de oxigênio nos tecidos impossibilitando a realização da troca gasosa e reduzindo o metabolismo celular, podendo causar acidose intracelular, dano vascular, inflamação local e morte celular, além da grande contribuição em alterações dos fatores de coagulação, aumentando ainda mais o risco de trombose e eventos que podem causar danos cardíacos. Esse fator que está sendo 
relacionado à lesão no miocárdio leva a apoptose e lesão das fibras musculares cardíacas por consequência do influxo de íons de cálcio (CABRAL et al., 2020).

\subsection{Tempestades de citocinas}

A tempestade de citocinas é uma resposta inflamatória demasiada frente à COVIDI9, onde há uma produção elevada de citocinas, como a IL-I, IL-2, IL-4, IL-6, ILıo, IL-I2, GCSF, IFN- $\gamma$ e TNF- $\alpha$. A IL-6 é considerada a mais importante, pois se mostra aumentada em pacientes graves infectados pela COVID-19, estando correlacionada a forma fatal da doença. Essa tempestade de citocinas leva a danos sistêmicos e consequentemente prejudica o tecido cardiovascular, além de causar permeabilidade vascular aumentada, dano epitelial alveolar e síndrome do desconforto respiratório agudo. $\mathrm{O}$ dano celular é causado devido à resposta inflamatória excessiva promovida pela grande quantidade de citocinas circulantes (CABRAL et al., 2020).

\subsection{Diagnóstico da covid-ı́ e biomarcadores que avaliam os danos cardíacos}

Seu diagnóstico é realizado através de exames laboratoriais que conseguem identificar a existência do vírus no organismo do paciente, por se tratar de uma doença recente, o diagnostico se torna importante, pois os sintomas da COVID-I9 são inespecíficos, podendo ser associados a outras patologias. Segundo a OMS, 2020, são utilizados dois métodos para o diagnóstico, a técnica padrão-ouro utilizada identifica o vírus por meio da constatação do RNA viral, nomeada de reação em cadeia da polimerase de transcrição reversa (RT-PCR), o outro teste é realizado pela identificação de anticorpos presente no organismo do paciente. A coleta do material para o exame deve ser feita em dias específicos após a aparição de sintomas levando em conta o tempo de incubação do vírus de 5 a 6 dias, pois esse também é um fator que pode interferir no resultado (WORLD HEALTH ORGANIZATION, 2020; UDUGAMA et al., 2020).

Em pacientes mais críticos, apresentando ou não comorbidades cardíaca, é indispensável à realização de exames para verificação dos parâmetros laboratoriais utilizados para avaliação da condição do paciente, sendo eles o hemograma apresentando uma linfopenia, exames de perfil de coagulação como tempo de tromboplastina parcial (TP) e tempo de tromboplastina parcial ativado (TTP), testes bioquímicos séricos incluindo 
função renal e hepática, creatina quinase, lactato desidrogenase e eletrólitos, interleucina-6 (IL-6), ferritina sérica e procalcitonina (TERPOS et al., 2020).

Pacientes que possuem a lesão cardíaca e se encontram em estado mais grave causado pela COVID-ı, é mais descrito na literatura a utilização de dois biomarcadores para acompanhar e avaliar o prognóstico, sendo a Troponina ultrassensível e o Dímero-D. Mais casos de pacientes que vieram a óbito estão associados a altos níveis de Troponina ultrassensível e o Dímero-D aumentado contribui para as respostas de citocinas próinflamatórias sistêmicas que são intermediários da aterosclerose, contribuindo diretamente para a suspensão da placa através da inflamação local, indução de fatores pró-coagulantes e alterações hemodinâmicas, que antecipam á isquemia e trombose. Esses biomarcadores não são os únicos utilizados para fazer o diagnóstico de uma lesão cardíaca, sendo realizado também eletrocardiograma de I2 derivações (ECG) (BRANDÃO; ANDRADE; FEITOSA, 2020; TERPOS et al., 2020).

\subsection{Tratamento}

O controle sobre a COVID-19 ainda possui limitações, pois não se encontra um tratamento especifico para a doença durante um surto. Os esforços tem se voltado para utilização de medicamentos já existentes e na criação da vacina. Quando a doença evolui para um estado grave, é dado início ao tratamento de suporte que é constituído por oxigenioterapia, tratamento conservador de fluidos, antimicrobianos empíricos e não se devem utilizar corticosteroides sistêmicos de forma rotineira. Quando se predomina o dano cardíaco é recomendado à iniciada de terapias de suporte sempre que oportuno, como antibióticos e anticoagulantes. (GUZIK et al., 2020; MINISTÉRIO DA SAÚDE, 2020).

Estão sendo utilizadas terapias antivirais para tentar impedir a replicação do vírus que incluem inibidores de protease, como liponovir/ritonavir, remdensivir e repressores imunológicos como cloroquina ou a hidroxicloroquina, porém ainda não se obteve resultados conclusivos com o uso desses medicamentos. Devido a tempestades de citocinas, que até então é apresentada por pacientes que possuem um agravamento mais acelerado, condutas de supressão imunológica e modulação imunológica têm sido experimentadas, pacientes com comprometimento cardíaco podem se beneficiar com essa abordagem. O Interferon beta (IFN- $\beta$ ), utilizado para tratamento de esclerose múltipla, aumenta a atividade das 
células $\mathrm{T}$ supressoras, diminuindo a produção de citocinas pró-inflamatórias, o que gera um benefício em pacientes com miocardite que desenvolvem disfunção sistólica ventricular esquerda (GUZIK et al., 2020).

\section{CONSIDERAÇÕES FINAIS}

O Sars-CoV-2, devido a ter rapidamente se alastrado para o mundo levando a uma pandemia, possui uma investigação crescente, sendo o desenvolvimento da infecção e seus mecanismos fisiológicos e patológicos cada vez melhor elucidados devido às inúmeras pesquisas que realizadas continuamente. Entretanto, ainda não existe uma estratégia clara de tratamento. Estudos evidenciam que a COVID-19, além de provocar danos respiratórios, acarretam também diversas complicações cardíacas. Principalmente através de lesão direta do vírus no tecido, utilizando a $\mathrm{ECA}_{2}$ como intermediário para entrada nas células. Biomarcadores como a troponina ultrassensível e Dímero-D demonstram-se bastante elevados em pacientes com lesão cardiovascular, levando a indicação de encaminhamento do paciente para UTI ou a óbito, podendo ser utilizados como ferramenta de diagnóstico precoce e prognóstico.

\section{REFERÊNCIAS BIBLIOGRÁFICAS}

BRANDÃO, S.C.S; ANDRADE, A.W; FEITOSA, A.D.M. Manual de condutas: COVID-19 e coração. I. Ed. Recife. 2020.

CABRAL, A.A; LEMOS, C.E.G; SOUZA, E.N; MOTA, L.R; RODRIGUES, M.R.B; LIMA, R.M; GADELHA, M.S.V. Patogênese do sistema cardiovascular em pacientes com covid-19. COVID-ı́ no Brasil Os Múltiplos Olhares da Ciência para Compreensão e Formas de Enfrentamento. C.:9, p.: 81-91. 2020.

CHEN, N.; ZHOU, M.; DONG, X.; QU, J.; GONG, F.; HAN, Y.; QIU, Y.; WANG, J.; LIU, Y.; WEI, Y.; XIA, J.; YU, T.; ZHANG, X.; ZHANG, L. Epidemiological and clinical characteristics of 99 cases of coronavirus pneumonia 2019 in Wuhan, China: a descriptive study. Lancet (Londres, Inglaterra) vol. 395,10223. 507-513. 2020. 
DAWEI W.; BINBIN W.; BO H.; CHANG H.; FANGFANG Z.; HUI X.; JING Z.;

XING L.; XINGHUAN W.; YAN Z.; YIRONG L.; YONG X.; ZHENSHUN Cheng; ZHIYONG P. Clinical Characteristics of 138 Hospitalized Patients With 2019 Novel Coronavirus-Infected Pneumonia in Wuhan, China. JAMA. 323 (II): I06I-I069. 2020.

GUZIK, T.J; MOHIDDIN, S.A; DIMARCO, A.; PATEL, V.; SAVVATIS, K.; MARELLI-BERG, F.M; MADHUR, M.S; TOMASZEWSKI, M.; MAFFIA, P.; D'ACQUISTO, F.; NICKLIN, S.A; MARIAN, A.J; NOSALSKI, R.; MURRAY, E.C; GUZIK, B.; BERRY, C.; LIL, B.; YANG, J.; ZHAO, F.; ZHI, L.; WANG, X.; LIU, L.; BI, Z.; ZHAO, Y. Prevalência e impacto de doenças metabólicas cardiovasculares em COVIDI9 na China. Clin Res Cardiol I09, 531-538. 2020.

GUO, T.; FAN, Y.; CHEN, M.; WU, X.; ZHANG, L.; HE, T.; WANG, H.; WAN, J.; WANG, X.; LU, Z. Cardiovascular Implications of Fatal Outcomes of Patients With Coronavirus Disease 2019 (COVID-19). JAMA Cardiol. 2020;5(7):8II-818. 2020.

MADJID M., ORLY V., SAFAVI-NAEINI P. SOLOMON, S.D. Potential Effects of Coronaviruses on the Cardiovascular System: A Review. JAMA Cardiol. 5 (7): 831840. 2020.

MCINTOSH, K. Coronavirus disease 2019 (COVID-19). UpToDate. March 31, 2020.

MENEZES, M.E. Diagnóstico laboratorial do coronavírus (SARS-CoV-2) causador da COVID-r9. Sociedade Brasileira de Análises Clínicas. 30. Março, 2020.

MINISTÉRIO DA SAÚDE. Protocolo de Tratamento do Novo Coronavírus (2019-nCoV). I a edição - publicação eletrônica. 2020 .

OMS. Painel da doença coronavírus da OMS (COVID-19). Genebra: Organização Mundial da Saúde, 2020. 
PASTRIAN-SOTO, G. Bases Genéticas y Moleculares del COVID-19 (SARS-CoV-2). Mecanismos de Patogénesis y de Respuesta Inmune. Int. J. Odontostomat., Temuco, v. I4, n. 3, p. 33I-337. 2020.

SUAIDEN, A.S. Responsabilidade de um farmacêutico em tempos de pandemia. Instituto de Ciência, Tecnologia e Qualidade. 03 Junho, 2020.

TERPOS E.; DIMOPOULOS M.A; ELALAMY I.; GEROTZIAFAS G.; KASTRITIS E.; NTANASIS-STATHOPOULOS I.; POLITOU M.; PSALTOPOULOU T.; SERGENTANIS T.N. Hematological findings and complications of COVID-19. American Journal of Hematology. April 13, 2020.

UDUGAMA, B; KOZLOWSK, H.N; MALEKJAHANI, A.; OSBORNE, M.; LI, V.Y.C; CHEN, H.; MUBAREKA, S.; GUBBAY, J.B; CHAN, W.C.W. Diagnosing COVID-rg: the disease and tools for detection. ACS nano, v. I4, n. 4, p. 3822-3835. 2020.

WORLD HEALTH ORGANIZATION. Diagnostic testing for SARS-CoV-2. II September, 2020 .

XIAOWEI, L; LIESU, M.; MANMAN, G.; SHEMIN, L.;; YIZHAO, P. Molecular immune pathogenesis and diagnosis of COVID-rg. Journal of Pharmaceutical Analysis io. p. 102-108. 2020.

ZHENG, Y.; MA, Y.; XIE, X.; ZHANG, J. COVID-rg and the cardiovascular system. Nature Reviews Cardiology. V. 17. P. 259. May 2020. 